\title{
Friedreich and dominant ataxias: quantitative differences in cerebellar dysfunction measurements
}

\author{
Audrey Tanguy Melac, ${ }^{1}$ Caterina Mariotti, ${ }^{2}$ Antoine Filipovic Pierucci, ${ }^{3}$ Paola Giunti, ${ }^{4}$ \\ Javier Arpa, ${ }_{1}^{5}$ Sylvia Boesch, ${ }^{6}$ Thomas Klopstock, ${ }^{7,8,9}$ Jennifer Müller vom Hagen, ${ }^{10,11}$ \\ Thomas Klockgether, ${ }_{1}^{12,13}$ Katrin Bürk, ${ }^{14,15}$ Jörg B Schulz, ${ }^{16}$ Kathrin Reetz, ${ }^{16}$ \\ Massimo Pandolfo, ${ }^{17}$ Alexandra Durr, ${ }^{18}$ Sophie Tezenas du Montcel, ${ }^{1,19,20}$ on behalf of \\ the EFACTS group
}

- Additional material is published online only. To view please visit the journal online (http://dx.doi.org/10.1136/ jnnp-2017-316964).

For numbered affiliations see end of article.

\section{Correspondence to} Dr Sophie Tezenas du Montcel, Département de Santé Publique, Unité de Biostatistiques et Information Médicale, Groupe Hospitalier Pitié - Salpêtrière, Paris 75651, France; sophie. tezenas@aphp.fr

Received 28 July 2017 Revised 11 November 2017 Accepted 19 November 2017 Published Online First 26 December 2017

\section{Check for updates}

To cite: Tanguy Melac A Mariotti C, Filipovic Pierucci A, et al. J Neurol Neurosurg Psychiatry 2018:89:559-565.

\section{ABSTRACT}

Background Sensitive outcome measures for clinical trials on cerebellar ataxias are lacking. Most cerebellar ataxias progress very slowly and quantitative measurements are required to evaluate cerebellar dysfunction.

Methods We evaluated two scales for rating cerebellar ataxias: the Composite Cerebellar Functional Severity (CCFS) Scale and Scale for the Assessment and Rating of Ataxia (SARA), in patients with spinocerebellar ataxia (SCA) and controls. We evaluated these scales for different diseases and investigated the factors governing the scores obtained. All patients were recruited prospectively.

Results There were 383 patients with Friedreich's ataxia (FRDA), 205 patients with SCA and 168 controls. In FRDA, 31\% of the variance of cerebellar signs with the CCFS and $41 \%$ of that with SARA were explained by disease duration, age at onset and the shorter abnormal repeat in the FXN gene. Increases in CCFS and SARA scores per year were lower for FRDA than for SCA (CCFS index: $0.123 \pm 0.123$ per year vs $0.163 \pm 0.179, \mathrm{P}<0.001$; SARA index: $1.5 \pm 1.2$ vs $1.7 \pm 1.7, P<0.001)$, indicating slower cerebellar dysfunction indexes for FRDA than for SCA. Patients with SCA2 had higher CCFS scores than patients with SCA1 and SCA3, but similar SARA scores. Conclusions Cerebellar dysfunction, as measured with the CCFS and SARA scales, was more severe in FRDA than in patients with SCA, but with lower progression indexes, within the limits of these types of indexes. Ceiling effects may occur at late stages, for both scales. The CCFS scale is rater-independent and could be used in a multicentre context, as it is simple, rapid and fully automated.

Trial registration number ClinicalTrials.gov: NCT02069509.

\section{INTRODUCTION}

There is clear need for scales for assessing cerebellar dysfunction for clinical trials on ataxia. It is crucial to capture small variations of cerebellar dysfunction for cerebellar diseases that progress slowly. The most frequent cerebellar diseases are Friedreich's ataxia (FRDA) and autosomal dominant spinocerebellar ataxias (SCAs) resulting from an expansion of CAG repeats in the SCA1, 2, 3 and 7 genes. ${ }^{1}$ FRDA is an autosomal-recessive disease consisting of progressive cerebellar and sensory ataxia, beginning around puberty. ${ }^{2}$ The European Friedreich's Ataxia Consortium for Translational Studies (EFACTS) was created in framework European framework (as part of the FP7 programme) and has assembled the necessary expertise for a fully translational research strategy on FRDA and its treatment. The European registry of patients with FRDA is an essential component of clinical studies, including observational studies and clinical trials. ${ }^{3}$

Several scales including clinical elements have been proposed for measuring FRDA severity and progression, including the International Cooperative Ataxia Rating Scale (ICARS) ${ }^{4}$ the Friedreich Ataxia Rating Scale (FARS) ${ }^{5}$ and the Scale for the Assessment and Rating of Ataxia (SARA). ${ }^{6}$ Performance-based scales have been successfully used in FRDA $^{7}$ and are less subject to inter-rater variability than clinical scales. This is a desirable feature given the multicentre context in which FRDA studies are performed. The Composite Cerebellar Functional Severity (CCFS) score is a quantitative performance-based scale validated for autosomal-dominant SCA in adults and children. ${ }^{8-10}$

The objectives of this study were to compare severity of the disease of both FRDA and SCA, to assess clinical utility of the SARA and CCFS scales for studies and to identify the factors governing the scores obtained for these scales. The results of this study will be important for the validation of these two scales for clinical trials.

\section{METHODS}

Within the EFACTS framework (http://www.e-facts. $\mathrm{eu}$, patients with FRDA were enrolled in a prospective, longitudinal study at 11 European centres.

\section{Patients}

Between October 2010 and June 2015, 605 adults and children over the age of 6 years were included in this study performed within the EFACTS network, including 383 patients (Aachen, $\mathrm{n}=27$; Bonn, $n=14$; Brussel, $n=27$; Innsbruck, $n=35$; London, $\mathrm{n}=60$; Madrid, $\mathrm{n}=47$; Marburg, $\mathrm{n}=5$; Milan, $\mathrm{n}=80$; Munich, $\mathrm{n}=32$; Paris, $\mathrm{n}=42$ and Tübingen, $n=14$ ) with both CCFS and SARA evaluations, together with information about age at onset (defined as age at the time of the first clinical 
Table 1 Characteristics of subjects per diagnosis at visit

\begin{tabular}{|c|c|c|c|c|}
\hline & FRDA $(n=383)$ & SCA $(n=205)$ & Controls $(n=168)$ & $P$ value* \\
\hline Women, N (\%) & $216(56 \%)$ & $103(50 \%)$ & $103(61 \%)$ & 0.0959 \\
\hline Age at examination, years, mean (SD) (minimum-maximum) & $33.1(14.6)(6-76)$ & $48.2(13.9)(15-83)$ & $21.4(16.6)(7-74)$ & $<0.0001$ \\
\hline Age at onset, years, mean (SD) (minimum-maximum) & $17.8(11.3)(1-65)$ & $36.6(12.9)(1-67)$ & - & $<0.0001$ \\
\hline Disease duration, years, mean (SD) (minimum-maximum) & $15.3(9.0)(1-49)$ & $11.7(8.5)(0-56)$ & - & $<0.0001$ \\
\hline CCFS, mean (SD) (minimum-maximum) & $1.225(0.158)(0.883-1.756)$ & $1.101(0.175)(0.661-1.680)$ & $0.843(0.045)(0.706-0.981)$ & $<0.0001$ \\
\hline SARA (range, 0-40), mean (SD) (minimum-maximum) & $18.3(8.4)(1.5-38)$ & $13.5(7.1)(0-32)$ & $0.8(1.0)(0-1)(n=18)$ & $<0.0001$ \\
\hline
\end{tabular}

${ }^{*} \mathrm{P}$ value of the comparison between all disease groups (ANOVA test or $\chi^{2}$ test).

ANOVA, analysis of variance; CCFS, Composite Cerebellar Functional Severity; FRDA, Friedreich's ataxia, SARA, Scale for the Assessment and Rating of Ataxia; SCA: dominant cerebellar ataxia.

symptoms) and disease duration (see online supplementary figure 1).

For an additional 199 patients with FRDA (Aachen, $\mathrm{n}=2$; Bonn, $\mathrm{n}=2$; Innsbruck, $\mathrm{n}=6$; London. $\mathrm{n}=85$; Madrid, $\mathrm{n}=29$; Marburg, $n=2$; Milan, $n=53$; Munich. $n=5$; Paris, $n=7$ and Tübingen, $n=8$ ) SARA scores and disease durations were available, but CCFS values were missing due a lack of availability of the device required for this test or the patient's limitations.

We also recruited 205 consecutive patients with dominant ataxias due to mutations of the SCA1, 2, 3 and 7 genes attending the Genetics Department and the National Reference Center for Rare Diseases of Pitié-Salpêtrière University Hospital in Paris. Age at onset, disease duration and SARA evaluation data were available for all these subjects.

Finally, 168 healthy control individuals (7-74 years of age) were also recruited in Milan and Paris.

For all individuals, information about the sex of the patient, age at examination and centre was available.

\section{Ethical standards}

Informed consent was obtained from patients or their authorised representatives (for patients under the age of 18 years or unable to confirm consent due to clinical impairment) before inclusion in the study, in accordance with the protocol for the EFACTS study or that for the SPATAX study (INSERM C10-41 and INSERM RBM 01-29). These studies are registered with ClinicalTrials.gov, number NCT02069509. This study was, therefore, performed in accordance with the ethical standards laid down in the 1964 Declaration of Helsinki and its subsequent amendments.

\section{Genetics}

Genetic testing (number of GAA-triplet-repeat expansions within the first intron of the frataxin gene or the existence of a point mutation) was performed for all patients with FRDA as described by the Laboratory of Experimental Neurology at the Université Libre de Bruxelles (Brussels, Belgium). ${ }^{11}$ For EFACTS, inclusion required a confirmed genetic diagnosis of FRDA. Patients with point mutations were excluded $(n=15)$. Genetic information for subjects with SCA was taken from their medical records.

\section{Clinical evaluation}

SARA is a semiquantitative scale developed for the assessment of functional impairments due to ataxia, with values from 0 (no ataxia) to 40 (most severe ataxia). It consists of eight items assessing stance, sitting, speech disturbance, finger chase, dysmetria, nose-finger test, tremor, fast alternating hand movements and heel-shin slide. ${ }^{6}$ The CCFS is a quantitative assessment initially developed and validated for comparisons of subjects with SCA with healthy controls.
It includes two functional tests for the dominant hand: the nine-hole pegboard test (time required to place dowels in nine holes) and the click test (time required to perform 10 finger-pointing cycles). ${ }^{8}$ As performance is age-dependent, the times required to perform the two tests are adjusted for age by calculating $\mathrm{Z}$-scores, which are then added together to give the CCFS score. ${ }^{10}$ An electronic device is used to acquire test times automatically and to calculate the final score. CCFS score is a quantitative score, independent of age, with higher values indicative of more severe cerebellar impairment. Both tests were administered by a trained clinician or assistant, during the EFACTS visits for subjects with FRDA and during routine annual follow-up visits for the other subjects.

\section{Statistical analysis}

Cerebellar dysfunction indexes were calculated by dividing the CCFS score (and the SARA score) by the disease duration to distinguish the impairment due to the disease itself from the impairment due to longer disease duration.

We compared population characteristics and severity scores between diagnoses, using the Pearson's $\chi^{2}$ test for qualitative variables or analysis of variance (ANOVA) for quantitative variables. Posthoc pairwise $t$-tests were performed for each significant ANOVA, with P values adjusted for multiple testing by Holm's method $\left(\mathrm{P}_{\mathrm{c}}\right)$. Differences in characteristics between subjects with and without available CCFS data were assessed by ANOVA. For subjects without available CCFS data, we compared those unable to perform the test with those for whom the test could not be performed for technical reasons.

Correlation between CCFS score, SARA score, age at onset, disease duration and the number of repeats of the shorter allele were assessed and are reported with a 95\% CI and P values.

We assessed the floor and ceiling effects of the SARA and CCFS scales, by analysing the relationship between CCFS and SARA scores by linear regression, with the addition of a quadratic term to the regression model and with non-linear models (sigmoid models) for each diagnosis. In the sigmoid model, we used the Gauss-Newton algorithm, with estimation by least squares, ${ }^{12}$ and the most parsimonious model was retained. The relationship between the SARA dysfunction index and the CCFS dysfunction index was studied in a similar manner. Possible confounding factors were taken into account by multivariate linear regression analysis, including age at onset, disease duration and short allele length, in the FRDA population.

Structural equation modelling (SEM) was used to check the statistical plausibility of our hypotheses concerning the links between variables, based on recommended association of fit indices ${ }^{13}$ : the standardised root mean square residual, for which values below 0.08 were considered to indicate a good fit, and the 
normed fit index, for which values above 0.96 were considered to indicate a good fit.

No imputation was performed for missing data. Data are expressed as means $\pm S D$ except for regression results, which are expressed as means \pm SE. Statistical analyses were carried out with R V.3.2.4 (R Foundation for Statistical Computing, Vienna, Austria) and SAS V.9.4 software for SEM. All tests were two-tailed and P values below 0.05 were considered statistically significant.

\section{RESULTS}

Clinical characteristics of subjects per diagnosis at visit are given in table 1.

Patients with FRDA were significantly younger than patients with SCA and older than controls (33.1 \pm 14.6 years

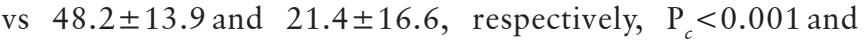
$\left.\mathrm{P}_{c}<0.001\right)$. They were also significantly younger at disease onset $\left(17.8 \pm 11.3\right.$ years vs $\left.36.6 \pm 12.9, \mathrm{P}_{c}<0.001\right)$. Disease duration was significantly shorter for patients with SCA than for patients with FRDA (11.7 \pm 8.5 years vs $15.3 \pm 9.0$, $\left.\mathrm{P}_{c}<0.001\right)$. The mean number of GAA repeats was 550 for

A

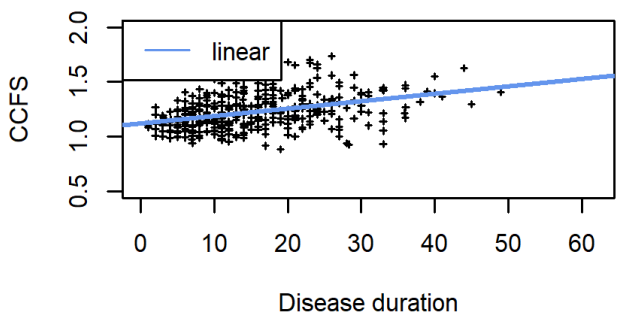

C

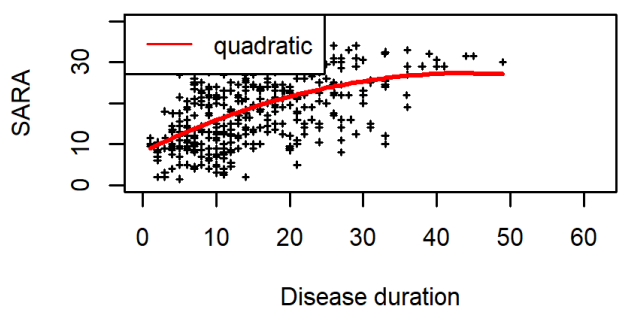

$\mathbf{E}$

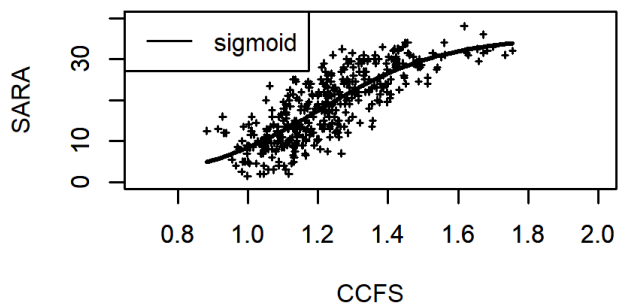

the shorter allele (range: 60-1200) and 882 for the longer allele (range: 150-1334). The mean number of expanded CAG repeats was 46 for SCA1 (range: 39-57), 40 for SCA2 (range: $35-48$ ), 72 for SCA3 (range: 56-78) and 48 for SCA7 (range: 42-62).

Comparison of the severity scores between disease groups Both CCFS and SARA scores differed between diseases, with these scores higher for patients with FRDA than for patients with SCA and controls (table 1: CCFS: $1.225 \pm 0.158$ vs $1.101 \pm 0.175$ and $0.843 \pm 0.045 \quad \mathrm{P}_{c}<0.001$ and $\mathrm{P}_{c}<0.001$; SARA: $18.3 \pm 8.4$ vs $13.5 \pm 7.0$ and $0.8 \pm 1.0, \mathrm{P}_{c}<0.001$ and $\left.\mathrm{P}_{c}<0.001\right)$, indicating more severe disease. After adjustment for disease duration, we used these scales to evaluate the cerebellar dysfunction. The CCFS and SARA cerebellar dysfunction indexes were smaller in FRDA than in patients with SCA (CCFS index: $0.123 \pm 0.123$ per year vs $0.163 \pm 0.179$, $\mathrm{P}<0.001$; SARA index: $1.5 \pm 1.2$ vs $1.7 \pm 1.7, \mathrm{P}<0.001$ ), indicating slower cerebellar dysfunction in FRDA than in SCA. Differences between genetic subtypes were detected in the population with SCA: patients with SCA2 had higher CCFS

B

Patients with SCA

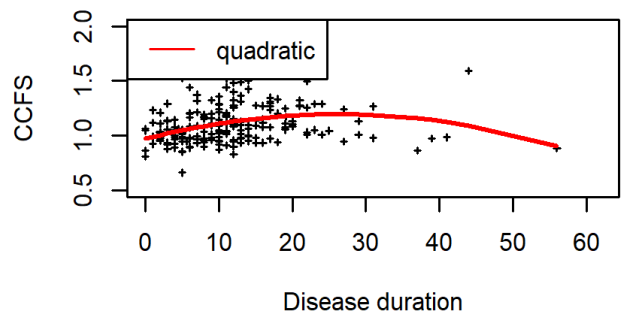

D

Patients with SCA

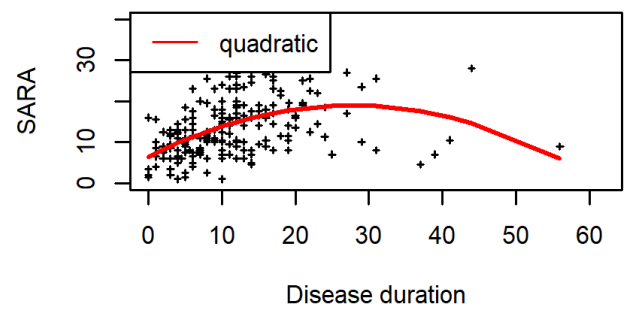

$\mathbf{F}$

Patients with SCA

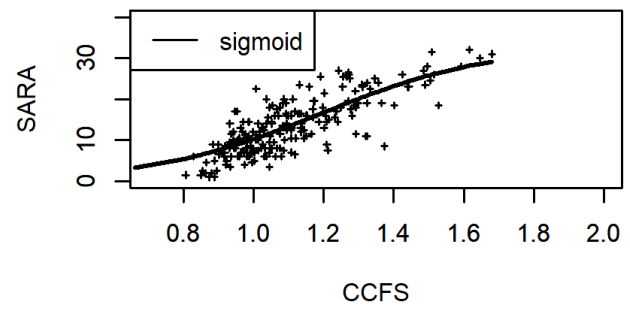

Figure 1 Relationship between CCFS score and disease duration (panels $A, B$ ), between SARA score and disease duration (panels C,D) and between SARA and CCFS scores (panels E,F), by diagnosis (FRDA or SCA). The relationship between CCFS and disease duration is linear while the relationship between SARA and disease duration is quadratic in patients with FRDA. In patients with SCA, both relationships are quadratic. The relationship between CCFS and SARA is sigmoid in both patients with FRDA and patients with SCA with a not linear relationship below a threshold of 10 and above a threshold of 30 for patients with FRDA and below 6 and above 26 in patients with SCA. CCFS, Composite Cerebellar Functional Severity; FRDA, Friedreich ataxia; SARA, Scale for the Assessment and Rating of Ataxia; SCA, dominant cerebellar ataxia. 
A

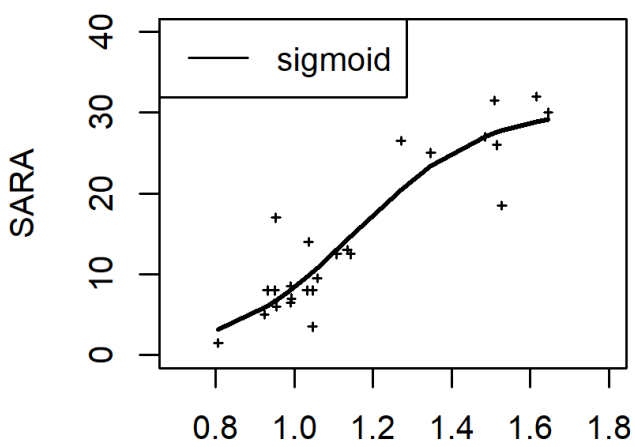

CCFS

C

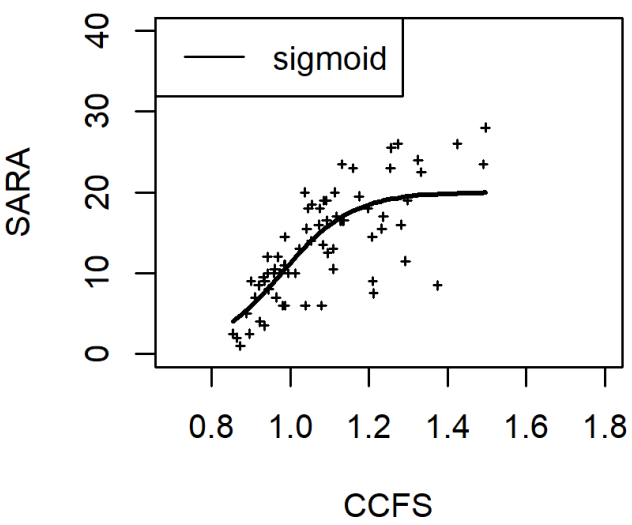

Patients with SCA2

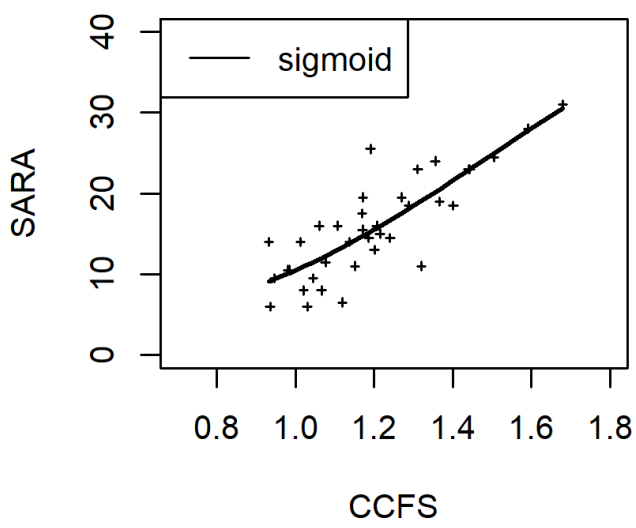

D

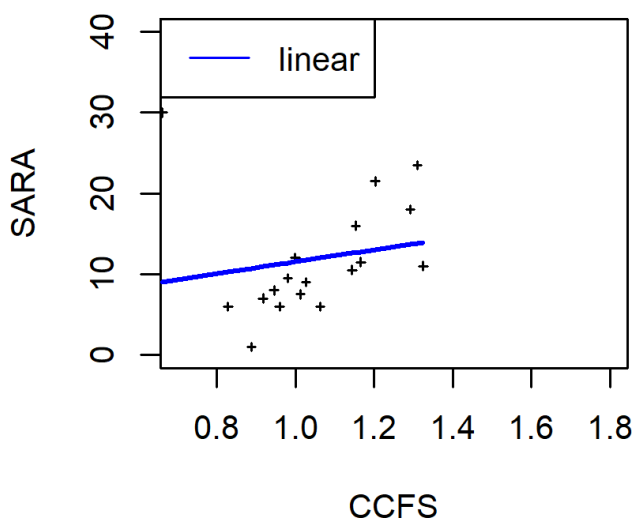

Figure 2 Relationship between CCFS and SARA scores, according to SCA subtype (panel A: SCA1, panel B: SCA2, panel C: SCA3, panel D: SCA7). The relationship between SARA and CCFS scores is not linear below a threshold of 10 and above a threshold of 24 for SCA1 and SCA2 and below a threshold of 7 and above a threshold of 16 for SCA3. CCFS, Composite Cerebellar Functional Severity; SARA, Scale for the Assessment and Rating of Ataxia; SCA, dominant cerebellar ataxia.

scores than patients with SCA1 and SCA3, but similar SARA scores (see online supplementary table 1 ).

Relationship between SARA score, CCFS score and disease duration in patients with FRDA and SCA

Regression analyses showed that CCFS and SARA increased (worsening) with disease duration in FRDA (figure 1A,C), but a ceiling effect was observed only for SARA (negative quadratic term, $\mathrm{P}=0.0045)$. This ceiling effect was weaker, but nevertheless present, in the subjects not undergoing CCFS testing included in the analysis (see online supplementary figure 2).

The scores on both scales increased in patients with SCA (figure 1B,D) and a ceiling effect was observed for disease duration (negative quadratic term, $\mathrm{P}=0.0001$ and $\mathrm{P}<0.0001$, respectively).

Furthermore, for regressions of SARA score against CCFS score, the best model for the patients with FRDA was a sigmoid model with both floor and ceiling effects (figure $1 \mathrm{E}, \mathrm{P}<0.0001$ ). The relationship between SARA and CCFS scores was not linear for SARA scores below 10 or above 24. For all patients with SCA considered together, the best model was a sigmoid model (figure $1 \mathrm{~F}, \mathrm{P}<0.0001$ ), also with thresholds of 10 and 24 . The sigmoid relationship was also the best fit for SCA1, 2 and 3 considered separately (figure 2A-C). For SCA7, the best relationship was linear, with 16 of the 18 SARA values lying between 6 and 24 (figure 2D).

\section{CCFS score and FRDA}

CCFS score was more closely correlated with disease characteristics (disease duration, age at onset and number of repeats in the shorter allele $(r=0.38(0.29$ to 0.46$), P<0.0001 ; r=-0.36$ $(-0.44$ to -0.27$), \mathrm{P}<0.0001$ and $\mathrm{r}=0.30 \quad(0.20$ to 0.38$)$, $\mathrm{P}<0.0001$, respectively) than with demographic characteristics, such as age at examination $(r=-0.04(-0.14$ to 0.06$)$, $\mathrm{P}=0.40)$. As expected, age at onset was closely correlated with the number of repeats $(\mathrm{r}=-0.65(-0.70$ to -0.59$), \mathrm{P}<0.001)$. We determined whether the relationship between the number of repeats in the shorter allele and the scores for the two scales was due to a direct relationship or a confounding effect of age at onset and/or disease duration, taking into account the pattern of correlations between variables, by performing SEM with all these variables (figure $3 \mathrm{~A}, \mathrm{~B}$ ). For both CCFS and SARA, scores were associated with the number of repeats in the shorter allele even after accounting for disease duration and age at onset. CCFS score, adjusted for the other covariates, increased by $0.28 \pm 0.06(\mathrm{P}<0.0001)$ for each additional 
A
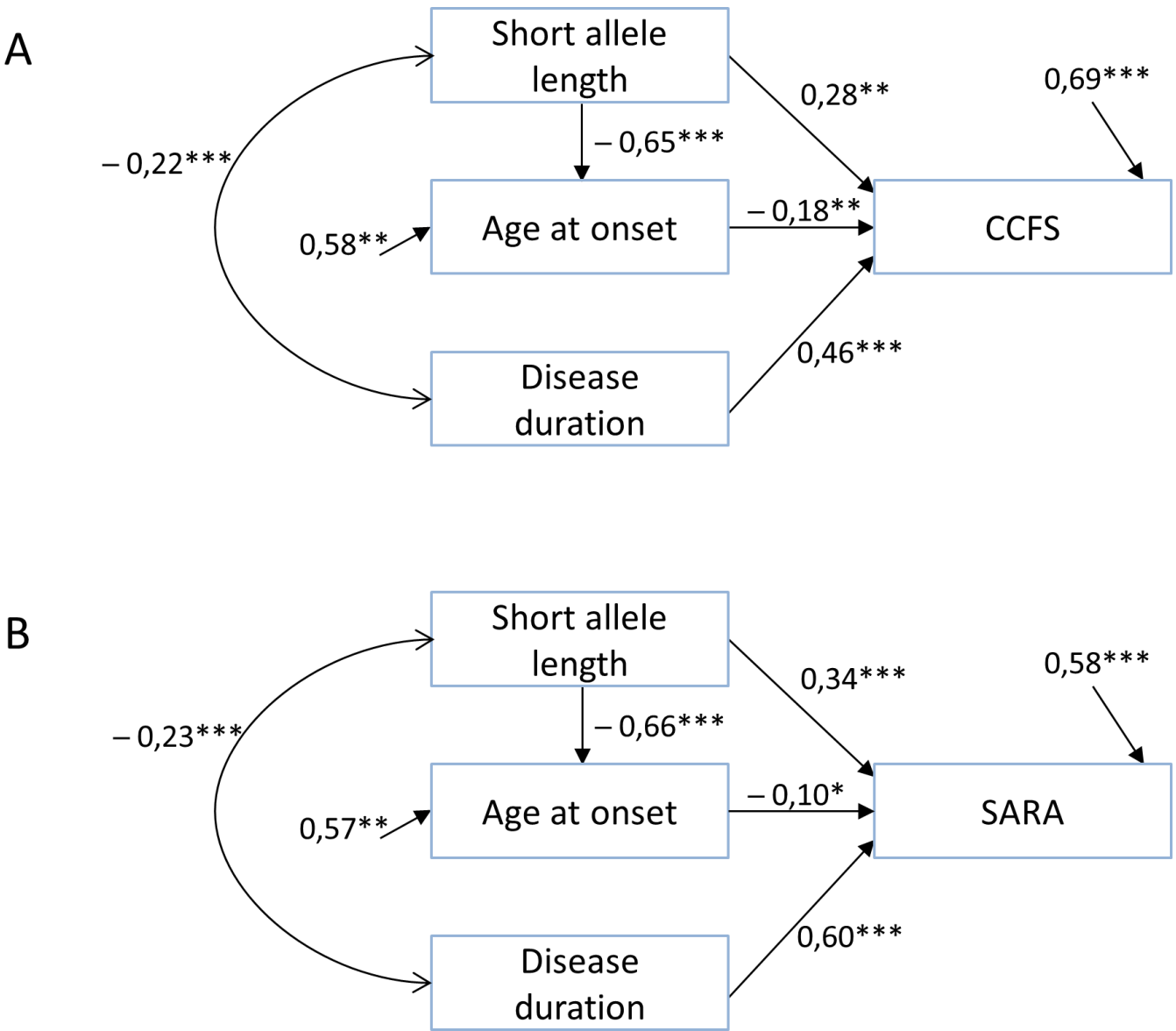

Figure 3 Final model of the influence of short allele length, age at onset and disease duration on scores in patients with FRDA. (A) CCFS score. The significant coefficients are indicated by asterisks. Value of fit indices: SRMR=0.045 and NFI=0.971. (B) SARA score. The significant coefficients are indicated by asterisks. Values of fit indices: $S R M R=0.048$ and $N F I=0.973$. Squares are representing the observed variables and arrows are representing the relationships between variables: single-headed arrows represent the impact of one variable on another and double-headed arrows represent correlations between pairs of variables. One-way arrows coming from outside are carrying the residual variance not explained by the model. CCFS, Composite Cerebellar Functional Severity; FRDA, Friedreich's ataxia; NFI, normed fit index; SARA, Scale for the Assessment and Rating of Ataxia; SRMR, standardised root mean square residual.

repeat and by $0.46 \pm 0.04(\mathrm{P}<0.0001)$ for each additional year of disease duration, whereas it decreased by $0.18 \pm 0.06$ for each additional year of age before onset $(\mathrm{P}<0.0001)$. Similarly, SARA score increased by $0.34 \pm 0.04(\mathrm{P}<0.0001)$ for each additional repeat and by $0.60 \pm 0.03(\mathrm{P}<0.0001)$ for each additional year of disease duration, whereas it decreased by $0.10 \pm 0.04(\mathrm{P}=0.017)$ for each year of age before onset. The models explained $31 \%$ of the variance for CCFS and $42 \%$ of the variance for SARA.

Characteristics of the populations with and without available CCFS score data

In total, 199 subjects underwent SARA testing, but not CCFS assessment. We found several differences between the patients who did and did not undergo CCFS testing. Patients who did not undergo CCFS testing had more severe disease: higher SARA scores $(28.8 \pm 7.8$ vs $18.3 \pm 8.4, \mathrm{P}<0.0001)$ and longer disease durations $(23.4 \pm 10.4$ vs $15.3 \pm 9.0, \mathrm{P}<0.0001)$ than patients who underwent CCFS testing. Those who were unable to perform the CCFS test had a longer disease duration $(26.5 \pm 9.5$ vs $19.6 \pm 10.1, \mathrm{P}<0.0001)$ and a higher SARA score $(32.3 \pm 5.0$ vs $24.4 \pm 8.5, \mathrm{P}<0.0001)$ than those for whom the test was not possible for technical reasons (see online supplementary table 2).

\section{DISCUSSION}

Cerebellar dysfunction, as assessed with the CCFS and SARA scales, was more severe in FRDA than in patients with SCA. However, after adjustment for disease duration, cerebellar function was found to be less impaired in FRDA than in patients with SCA, particularly for patients with SCA1 and 2, who are more prone to cerebellar dysfunction than patients with afferent ataxia. Thus the differences observed at inclusion are more explained by the disease duration than by the severity of the patients. These results should be confirmed with longitudinal data. These adjustments on disease duration have well-known limitations, especially for shorter disease durations and the extremes of disability: patients with a lower score and a shorter disease duration will have the same indexes as patients with a higher score and a longer disease duration.

SARA scores followed a linear trend between 10 and 24, with a floor and a ceiling in both patients with FRDA and patients with SCA. There are several possible explanations for this. First, SARA is a clinical assessment and is, thus, bounded, whereas the CCFS is unbounded. Second, the most severely affected patients may not have performed CCFS, thereby introducing a bias, although $27 \%$ of the subjects 
undergoing CCFS testing had a SARA score above 24. The large number of missing CCFS data requires further investigation. Third, the CCFS is an objective scale whereas SARA is a clinical scale that is more subjected, despite being semiquantitative and standardised. SARA score may be more variable in patients with more extensive neurological signs (pyramidal, extrapyramidal and so on). Finally, 21\% of those with a CCFS score $<10$ were children. Only the CCFS was validated in children. ${ }^{10}$ The CCFS and SARA scales may therefore assess dysfunction differently, and they may be complementary.

For disease duration, the ceiling effect of SARA ${ }^{14}$ and of other clinical rating scales, including ICARS and FARS, ${ }^{15} 16$ has already been reported for patients with FRDA. The ceiling effect of SARA and CCFS observed in patients with FRDA and SCA with long disease durations may reflect an underperformance of these scales for the most severe disease stages or a slowing of the biological progression of the disease. ${ }^{717}$ If confirmed in longitudinal studies, this ceiling effect of both scales at late stages has implications for the follow-up of the most severely affected FRDA subjects, particularly in terms of the potential use of these scales in clinical trials. ${ }^{18} 19$ And it adds a further bias to the indexes dividing the scores by the disease durations. There is no consensus method for determining progression of cerebellar dysfunction in patients with ataxia. There is a need for a powerful method to detect different rates of disease progression to assess the relationship between disability and disease duration and help guide treatment decisions.

The effect of the number of repeats on age at onset and on phenotypic severity has been reported elsewhere. ${ }^{20}$ Our study confirmed these findings and showed that the two scales gave similar results, with independent effects of both age at onset and disease duration in addition to short allele length, even when the complex correlations between variables were taken into account with an adapted model. SEM findings depend on the hypothetical path and, in this study, we confirmed the existence of a direct effect of age at onset and disease duration and direct and indirect effects of short allele length on both scales. However, these factors accounted for only a moderate proportion of the variance for the scores on these scales.

\footnotetext{
Author affiliations

'Department of Biostatistics and Medical Informatics, AP-HP, Groupe Hospitalier Pitié-Salpêtrière Charles-Foix, Paris, France

${ }^{2}$ Unit of Genetics of Neurodegenerative and Metabolic Diseases, Fondazione Istituto di Ricovero e Cura a Carattere Scientifico, Istituto Neurologico Carlo Besta, Milano, Italy

${ }^{3}$ URCEco and Santé publique, Assistance Publique Hopitaux de Paris, Paris, France ${ }^{4}$ Department of Molecular Neuroscience, UCL Institute of Neurology, London, UK ${ }^{5}$ Department of Neurology, Instituto de Investigación Hospital Universitario La Paz, Madrid, Spain

${ }^{6}$ Department of Neurology, Medical University Innsbruck, Innsbruck, Austria ${ }^{7}$ Department of Neurology, Friedrich-Baur-Institute, University of Munich, Munich, Germany

${ }^{8}$ German Center for Neurodegenerative Diseases (DZNE), Munich, Germany

${ }^{9}$ Munich Cluster for Systems Neurology (SyNergy), Munich, Germany

${ }^{10}$ Department of Neurodegenerative Diseases, Hertie Institute for Clinical Brain

Research, University of Tübingen, Tuebingen, Germany

${ }^{11}$ German Center for Neurodegenerative Diseases (DZNE), Tübingen, Germany

${ }^{12}$ Department of Neurology, University of Bonn, Bonn, Germany

${ }^{13}$ German Center for Neurodegenerative Diseases (DZNE), Bonn, Germany

${ }^{14}$ Department of Neurology, Philipps Universität Marburg, Marburg, Germany

${ }^{15}$ Paracelsus-Elena-Klinik, Kassel, Germany

${ }^{16}$ Department of Neurology, Center for Rare Diseases, Clinical Trial Centre, JARABRAIN Institute Molecular Neuroscience and Neuroimaging, Forschungszentrum Jülich GmbH and RWTH Aachen University, Aachen, Germany

${ }^{17}$ Department of Neurology, Hôpital Erasme, Bruxelles, Belgium
}

${ }^{18}$ Department of Genetics, ICM Institut du Cerveau et de la Moelle and APHP, University Hospital Pitié-Salpêtrière, Paris, France

${ }^{19}$ Sorbonne Universités, UPMC Univ Paris 06 UMR_S1136, Paris, France ${ }^{20}$ INSERM UMR_S 1136, Institut Pierre Louis d'Epidémiologie et de Santé Publique Paris, France

Acknowledgements We warmly thank all the subjects for participating in the study

Collaborators Affiliation of the EFACTS Coinvestigators: M Panzeri, MD (Unit of Genetics of Neurodegenerative and Metabolic Diseases, Fondazione Istituto di Ricovero eCura a Carattere Scientifico, Istituto Neurologico Carlo Besta, Milan, Italy, Site Investigator); M H Parkinson MBBS (Department of Molecular Neuroscience, UCL Institute of Neurology, London, UK, Site Investigator); I Sanz-Gallego, MD (Reference Unit of Hereditary Ataxias and Paraplegias, Department of Neurology, Instituto de Investigación Sanitaria, Hospital Universitario La Paz, Madrid, Spain, Site Investigator); W Nachbauer, MD (Department of Neurology, Medical University Innsbruck, Austria, Site Investigator); I Karin, MD (Friedrich-Baur-Institute, Department of Neurology, University of Munich, Germany, Site Investigator); C Depondt, MD (Laboratory of Experimental Neurology, Université Libre de Bruxelles, Brussels, Belgium, Site Investigator); L Schoels, MD PhD (Centre for Neurology and Hertie Institute for Clinical Brain Research, University of Tübingen, Tübingen, Germany; German Center for Neurodegenerative Diseases (DZNE), Tübingen, Germany, Site Investigator); I Giordano, MD (Department of Neurology, University Hospital of Bonn, Bonn, Germany; German Center for Neurodegenerative Diseases (DZNE), Tübingen, Germany, Site Investigator); L Nanetti, MD (Unit of Genetics of Neurodegenerative and Metabolic Diseases, Fondazione Istituto di Ricovero eCura a Carattere Scientifico, Istituto Neurologico Carlo Besta, Milan, Italy, Site Investigator); A Castaldo, MS (Unit of Genetics of Neurodegenerative and Metabolic Diseases, Fondazione Istituto di Ricovero e Cura a Carattere Scientifico, Istituto Neurologico Carlo Besta, Milan, Italy, Site Investigator), A Eigentler, MD, PhD (Medical University Innsbruck, Department of Neurology, Innsbruck, Austria, Site Investigator).

Contributors All authors give final approval of the version to be published and take responsibility for the conduct of the research. ATM: design, execution, review and critique of the statistical analysis, writing of the first draft, review and critique of the manuscript. CM, PG, JA, SB, TK, JMvH, TK, KB, JBS, KR, MP: conception, organisation and execution of the research project, review and critique of the manuscript. AFP: design of the statistical analysis, review and critique of the manuscript. AD: conception, organisation and execution of the research project, obtaining of the funding, review and critique of the statistical analysis, review and critique of the manuscript. STdM: conception of the research project, design, review and critique of the statistical analysis, review and critique of the manuscript.

Funding Projects EFACTS (UE FP7-HEALTH-2009/contract no. E10015DD) and Programme Hospitalier de Recherche Clinique AOM03059 (contract no. R05129DD) to $C M, P G, S B, J B S, K R, M P$ and $A D$.

Competing interests $C M, P G, J A, T K, J M v H, T K, K R$ have nothing to disclose. AFP has worked for the Health Economics and Health Policy Research Unit of Greater Paris University Hospitals. SB reports personal fees from Gruenenthal, AbbVie, Ipsen and Allergan, outside the submitted work. KB reports grants from University of Aachen/EU, during the conduct of the study; grants from Actelion, Pfizer and CHDI; personal fees from Desitin, Medtronic, outside the submitted work. JBS reports grants from 7th Framework of the EU; Funding of the "European Friedreich Ataxia Consortium for Translational Studies (EFACTS), during the conduct of the study; he serves on scientific advisory boards for Lundbeck, TEVA, Novartis and Lilly and have received funding for travel and speaker honoraria from GlaxoSmithKline, Merz Pharmaceuticals, Medical Tribune, Lundbeck, Pfizer, Boehringer and Bayer and has received research support from the BMBF, DFG and the EU. MP reports grants and personal fees from Voyager Therapeutics, personal fees from Apopharma, grants and personal fees from Biomarin, outside the submitted work. AD partly has nothing to disclose. STdM reports personal fees from Boston Scientific, grants from Agence Nationale de la Recherche, outside the submitted work.

Ethics approval Local ethic committees of each participating centre.

Provenance and peer review Not commissioned; externally peer reviewed.

(C) Article author(s) (or their employer(s) unless otherwise stated in the text of the article) 2018. All rights reserved. No commercial use is permitted unless otherwise expressly granted.

\section{REFERENCES}

1 Pulst SM. Ataxia rating scales in the balance. Nat Clin Pract Neurol 2007;3:119.

2 Schulz JB, Boesch S, Bürk K, et al. Diagnosis and treatment of Friedreich ataxia: a European perspective. Nat Rev Neurol 2009;5:222-34.

3 Reetz K, Dogan I, Costa AS, et al. Biological and clinical characteristics of the European Friedreich's Ataxia Consortium for Translational Studies (EFACTS) cohort: a cross-sectional analysis of baseline data. Lancet Neurol 2015;14:174-82. 
4 Trouillas P, Takayanagi T, Hallett M, et al. International cooperative ataxia rating scale for pharmacological assessment of the cerebellar syndrome. J Neuro/ Sci 1997;145:205-11.

5 Lynch DR, Farmer JM, Tsou AY, et al. Measuring Friedreich ataxia: complementary features of examination and performance measures. Neurology 2006;66:1711-6.

6 Schmitz-Hübsch T, du Montcel ST, Baliko L, et al. Scale for the assessment and rating of ataxia: development of a new clinical scale. Neurology 2006;66:1717-20.

7 Friedman LS, Farmer JM, Perlman S, et al. Measuring the rate of progression in Friedreich ataxia: implications for clinical trial design. Mov Disord 2010;25:426-32

8 du Montcel ST, Charles P, Ribai P, et al. Composite cerebellar functional severity score: validation of a quantitative score of cerebellar impairment. Brain 2008;131:1352-61.

9 Tezenas du Montcel S, Charles P, Goizet C, et al. Factors influencing disease progression in autosomal dominant cerebellar ataxia and spastic paraplegia. Arch Neurol 2012;69:500-8.

10 Filipovic Pierucci A, Mariotti C, Panzeri M, et al. Quantifiable evaluation of cerebellar signs in children. Neurology 2015;84:1225-32.

11 Pandolfo M. Friedreich ataxia: detection of GAA repeat expansions and frataxin point mutations. Methods Mol Med 2006;126:197-216.

12 Bates DM, Watts DG, eds. Nonlinear regression analysis and its applications. New York: John Wiley \& Sons, 1998.
13 Hooper D, Coughlan J, eds. Structural equation modelling: guidelines for determining model fit: Dublin Institute of Technology, Electronic Journal of Business Research Methods, 2008.

14 Marelli C, Figoni J, Charles P, et al. Annual change in Friedreich's ataxia evaluated by the Scale for the Assessment and Rating of Ataxia (SARA) is independent of disease severity. Mov Disord 2012;27:135-9.

15 Tai G, Corben LA, Gurrin L, et al. A study of up to 12 years of follow-up of Friedreich ataxia utilising four measurement tools. J Neurol Neurosurg Psychiatry 2015;86:660-6.

16 Ribaï P, Pousset F, Tanguy ML, et al. Neurological, cardiological, and oculomotor progression in 104 patients with Friedreich ataxia during long-term follow-up. Arch Neurol 2007;64:558-64.

17 Chan E, Charles P, Ribai P, et al. Quantitative assessment of the evolution of cerebellar signs in spinocerebellar ataxias. Mov Disord 2011;26:534-8.

18 Jacobi H, du Montcel ST, Bauer P, et al. Long-term disease progression in spinocerebellar ataxia types 1, 2, 3, and 6: a longitudinal cohort study. Lancet Neurol 2015;14:1101-8.

19 Reetz K, Dogan I, Hilgers RD, et al. Progression characteristics of the European Friedreich's Ataxia Consortium for Translational Studies (EFACTS): a 2 year cohort study. Lancet Neurol 2016;15:1346-54.

20 Dürr A, Cossee M, Agid Y, et al. Clinical and genetic abnormalities in patients with Friedreich's ataxia. N Engl J Med 1996;335:1169-75. 